\title{
Evidence for Supersymmetry in the Random-Field Ising Model at $D=5$
}

\author{
Nikolaos G. Fytas, ${ }^{1}$ Víctor Martín-Mayor, ${ }^{2,3}$ Giorgio Parisi, ${ }^{4}$ Marco Picco, ${ }^{5}$ and Nicolas Sourlas ${ }^{6}$ \\ ${ }^{1}$ Applied Mathematics Research Centre, Coventry University, Coventry CV1 5FB, United Kingdom \\ ${ }^{2}$ Departamento de Fúsica Téorica I, Universidad Complutense, 28040 Madrid, Spain \\ ${ }^{3}$ Instituto de Biocomputacíon y Física de Sistemas Complejos (BIFI), 50009 Zaragoza, Spain \\ ${ }^{4}$ Dipartimento di Fisica, Sapienza Università di Roma, \\ P.le Aldo Moro 2, 00185 Rome, Italy and INFN, Sezione di Roma I, \\ IPCF - CNR, P.le A. Moro 2, 00185 Rome, Italy \\ ${ }^{5}$ Laboratoire de Physique Théorique et Hautes Energies, UMR7589, \\ Sorbonne Université et CNRS, 4 Place Jussieu, 75252 Paris Cedex 05, France \\ ${ }^{6}$ Laboratoire de Physique Théorique de l'Ecole Normale Supérieure \\ (Unité Mixte de Recherche du CNRS et de l'Ecole Normale Supérieure, \\ associée à l'Université Pierre et Marie Curie, PARIS VI) 24 rue Lhomond, 75231 Paris Cedex 05, France
}

(Dated: June 6, 2019)

\begin{abstract}
We provide a non-trivial test of supersymmetry in the random-field Ising model at five spatial dimensions, by means of extensive zero-temperature numerical simulations. Indeed, supersymmetry relates correlation functions in a $D$-dimensional disordered system with some other correlation functions in a $D-2$ clean system. We first show how to check these relationships in a finite-size scaling calculation, and then perform a high-accuracy test. While the supersymmetric predictions are satisfied even to our high-accuracy at $D=5$, they fail to describe our results at $D=4$.
\end{abstract}

PACS numbers: $05.50 .+\mathrm{q}, 75.10 . \mathrm{Nr}, 02.60 . \mathrm{Pn}, 75.50 . \mathrm{Lk}$

Introduction.- The suggestion 1 that the randomfield Ising model (RFIM) at the critical point [2 4] obeys supersymmetry came as a major surprise in Theoretical Physics. One of the implications of supersymmetry is dimensional reduction [5, 6]: the critical exponents of a disordered system at space dimension $D$ and those of a pure (i.e. non-disordered) system at dimension $D-2$ coincide. Let us remark that dimensional reduction is a consequence of [1, 7], but not necessarily equivalent to, supersymmetry.

However, in spite of its power and elegance, it was soon clear that the applicability of supersymmetry is problematic. The original argument [1] was based on the study of the solutions of the stochastic Landau-Ginsburg equations in the presence of a random magnetic field. Unfortunately, the crucial assumption of uniqueness of the solution of these equations [1] (which holds at all orders in perturbation theory), fails beyond perturbation theory. In fact, it was immediately clear that in the RFIM the predicted dimensional reduction is absent at low dimensions (but not for branched polymers 8 , where dimensional reduction has been mathematically proven 9 11): the RFIM has a ferromagnetic phase at $D=3[12,13$, while the $D=1$ pure Ising model has no transition. Non-perturbative effects (e.g. bound-states in replica space [14 17]) are obviously important in $D=3$. Yet, their relevance for $D>3$ (specially upon approaching the presumed upper critical dimension $D_{\mathrm{u}}=6$ ) is unclear. If we consider the case of $D=6-\epsilon$, different scenarios are possible, as listed below:

1. Nonperturbative effects could destroy supersymmetry at a finite order in the $\epsilon$ expansion or, even worse, at $D=6$.
2. Violations of supersymmetry might be exponentially small $\sim \exp (-A / \epsilon)$ (see e.g. Refs. [18, 19; the computation of $A$ is still an unsolved problem).

3. Supersymmetry has been suggested to be exact but only for $D>D_{\text {int }} \approx 5.120-22$. For $D<D_{\text {int }}$ the supersymmetric fixed point becomes unstable with respect to non-supersymmetric perturbations.

In order to discriminate among these three scenarios, we need accurate simulations aimed to test some of the many predictions of supersymmetry. In the last few years, the development of a powerful panoply of simulation and statistical analysis methods 2325$]$ set the basis for a fresh revision of the problem. Great emphasis was made on the anomalous dimensions $\eta$ and $\bar{\eta}$ related to the decay of the connected and disconnected correlations functions, respectively [see Eq. (2)]. Supersymmetry predicts $\eta=\bar{\eta}$ (moreover, the $D$-dimensional RFIM $\eta=\bar{\eta}$ are predicted to be equal to the anomalous dimension of the pure Ising model in dimension $D-2$ ). Extensive numerical simulations at zero temperature showed that these relations fail at $D=322$ and $D=4$ [25], but they are valid with good accuracy at $D=5$ [26]. These numerical results suggest that supersymmetry may be really at play at $D=5$. We should mention as well a recent work using conformal boostrap [27, where it was found that dimensional reduction holds in the RFIM for $D \geq 5$.

The predictions of supersymmetry go further beyond those regarding the critical exponents: they involve both finite volume effects and high-order correlations functions. Here, we will show that several non-trivial supersymmetry predictions hold at $D=5$ to a very high numerical accuracy. This is the first direct confirmation 
that supersymmetry holds in the RFIM at high dimensions. As a consistency check, we show that the same relations are definitively not-satisfied at $D=4$.

Simulation setup. - The Hamiltonian of the RFIM is

$$
\mathcal{H}=-J \sum_{<x y>} S_{x} S_{y}-\sum_{x} h_{x} S_{x}
$$

with the spins $S_{x}= \pm 1$ on a hypercubic lattice in $D$ dimensions with nearest-neighbor ferromagnetic interactions and $h_{x}$ independent random magnetic fields with zero mean and variance $\sigma^{2}$. Given our previous universality confirmations [28, we have restricted ourselves to normal-distributed $h_{x}$. We work directly at zero temperature 2933 because the relevant fixed point of the model lies there 34 36. The system has a ferromagnetic phase at small $\sigma$, that, upon increasing the disorder, becomes paramagnetic at the critical point $\sigma_{\mathrm{c}}$. Here, we work directly at $\sigma_{\mathrm{c}}$, namely at $6.02395 \approx \sigma_{\mathrm{c}}(D=5)[26$ ] and at $4.17749 \approx \sigma_{\mathrm{c}}(D=4)$ 25].

We consider two correlation functions, namely the connected and disconnected propagators, $C_{x y}^{(\text {con })}$ and $C_{x y}^{(\text {dis })}$ :

$$
C_{x y}^{(\text {con })} \equiv \frac{\partial \overline{\left\langle S_{x}\right\rangle}}{\partial h_{y}}, C_{x y}^{(\text {dis })} \equiv \overline{\left\langle S_{x}\right\rangle\left\langle S_{y}\right\rangle},
$$

where the $\langle\cdots\rangle$ are thermal mean values as computed for a given realization, a sample, of the random fields $\left\{h_{x}\right\}$. Over-line refers to the average over the samples.

For each of these two propagators, we scrutinize the second moment correlation lengths [37, as adapted to our geometrical setting. In particular, our chosen geometry is an elongated hypercube with periodic boundary conditions and linear dimensions $L_{x}=L_{y}=L_{z}=L$ and $L_{t}=L_{u}=R L\left(\right.$ at $D=4$ we chose $L_{x}=L_{y}=L$ and $\left.L_{z}=L_{t}=R L\right)$ with aspect ratio $R \geq 1$. In fact, the supersymmetric identities that we will check in the critical region hold in the limit $R \rightarrow \infty$, which should be taken before the standard thermodynamic limit.

We simulated lattice sizes in the range $L=4-14$ at $D=5(L=4-28$ at $D=4)$ and aspect ratios $1 \leq R \leq$ 5. Additional simulations for $R=10$ and $L \leq 10$ were performed at both $5 \mathrm{D}$ and $4 \mathrm{D}$ for consistency reasons. For each pair of $(L, R)$-values we computed ground states for $10^{5}$ disorder samples. Our simulations and analysis closely follows the methodology outined in our previous works at $D=3$ and 4 [23, 25] (for full technical details see Ref. 24]).

Supersymmetric predictions. - Let us consider a point in the $5 \mathrm{D}$ lattice, $\mathbf{r}=(\mathbf{x}, \mathbf{u})$ where $\mathbf{x}=(x, y, z)$ refers to the first three cartesian coordinates, while $\mathbf{u}=(t, u)$. In a similar vein, for the $4 \mathrm{D}$ case, we split $\mathbf{r}=(x, y, z, t)=$ $(\mathbf{x}, \mathbf{u})$ as $\mathbf{x}=(x, y)$ and $\mathbf{u}=(z, t)$. The supersymmetric predictions (see [7, 38, 40] and Appendix A for a more paused exposition) are particularly simple for disconnected correlation functions:

$$
C_{\mathbf{x}_{1}, \mathbf{u} ; \mathbf{x}_{2}, \mathbf{u}}^{\text {dis),D }}=\mathcal{Z} G_{\mathbf{x}_{1} ; \mathbf{x}_{2}}^{\mathrm{Ising}, D-2},
$$

where $G$ is the pure Ising model correlator, and $\mathcal{Z}$ is a position independent normalization constant that will play no role (see below). Note that the left-hand side depends on both linear dimensions, $L$ and $R L$, while the right-hand side depends only on $L$. Therefore, we must carefully consider under which conditions Eq. (3) is expected to hold. In a more conventional study, one would require an hierarchy of length scales $L R \gg L \gg \xi \gg 1$ (recall that $\xi$ is the correlation length), while we demand for the $D-2$ Euclidean distance $\left\|\mathbf{x}_{1}-\mathbf{x}_{\mathbf{2}}\right\| / \xi \sim 1$. We shall put under stress Eq. (3) by demanding it to hold as well in the finite-size scaling regime

$$
L R \gg L \sim \xi \gg 1,\left\|\mathbf{x}_{1}-\mathbf{x}_{\mathbf{2}}\right\| / \xi \sim 1 .
$$

These preliminaries lead us to consider a $D-2$ Fourier transform in the $D$-dimensional RFIM

$$
\hat{C}_{\mathbf{k}}^{(\text {dis }), \mathrm{D}}=\frac{1}{L^{D-2}} \sum_{\mathbf{x}_{1}, \mathbf{x}_{2}} \mathrm{e}^{\mathrm{i}\left(\mathbf{x}_{1}-\mathbf{x}_{2}\right) \cdot \mathbf{k}} \overline{\left\langle S_{\mathbf{x}_{1}, \mathbf{u}}\right\rangle\left\langle S_{\mathbf{x}_{2}, \mathbf{u}}\right\rangle} .
$$

Note that the $\mathbf{u}$-dependence vanishes due to the disorderaverage (hence we average over $\mathbf{u}$ in order to gain statistics). We then compute the second-moment correlation length from the ratio of $\hat{C}_{\mathbf{k}}^{(\mathrm{dis}), \mathrm{D}}$ at $\mathbf{k}=\mathbf{0}$ and $\mathbf{k}_{\min }=(2 \pi / L, 0,0)$ 37] $\left[\mathbf{k}_{\min }=(2 \pi / L, 0)\right.$ for $\left.D=4\right]$. The important observation is that, because the constant $\mathcal{Z}$ in the r.h.s. of Eq. (3) cancels when computing the ratio, the dimensionless ratio $\xi^{\text {(dis) }} / L$ as computed in the $D$-dimensional RFIM coincides with $\xi / L$ as computed in the $D-2$ Ising model. This equality holds if $\xi^{\text {(dis) }} / L$ is computed precisely at the critical point $\sigma_{\mathrm{c}}$ and if the thermodynamic limit is taken under conditions (4).

If we now consider the four-body disconnected correlation function, supersymmetry predicts a relation analogous to Eq. (3) (the normalization in the r.h.s changes to $\left.\mathcal{Z}^{2}\right)$, so we may compute as well a $(D-2)$-dimensional $U_{4}$ parameter,

$$
M_{\mathbf{u}}=\sum_{\mathbf{x}} S_{\mathbf{x}, \mathbf{u}}, U_{4}=\overline{\left\langle M_{\mathbf{u}}^{4}\right\rangle} /{\overline{\left\langle M_{\mathbf{u}}^{2}\right\rangle}}^{2},
$$

that is predicted to coincide with that of the critical $D-2$ Ising model (under the same condition discussed above for $\left.\xi^{\text {(dis) }} / L\right)$. Again, we improve our statistics by averaging both $\overline{\left\langle M_{\mathbf{u}}^{4}\right\rangle}$ and $\overline{\left\langle M_{\mathbf{u}}^{2}\right\rangle}$ over $\mathbf{u}$.

We finally address the supersymmetric predictions for the connected correlation function. It is convenient to consider the correlation functions $K$ defined as

$$
K_{\mathbf{x}_{1} ; \mathbf{x}_{2}}=\sum_{\mathbf{u}} C_{\mathbf{x}_{1}, \mathbf{0} ; \mathbf{x}_{2}, \mathbf{u}}^{(\text {con }} .
$$

The Ward identity for supersymmetry 38, implies, see Appendix $\mathrm{B}$, that the second-moment correlation length $\xi_{\sigma-\eta}^{(\mathrm{con})}$ computed from $K$ [4] is equal to the disconnected correlations length. This prediction $\xi_{\sigma-\eta}^{(\text {con })}=\xi^{(\text {dis })}$ does not make direct reference to dimensional reduction.

Results. - Let us start by recalling in Table I the $(D-$ $2)=2,3$ universal quantities from the pure Ising model 


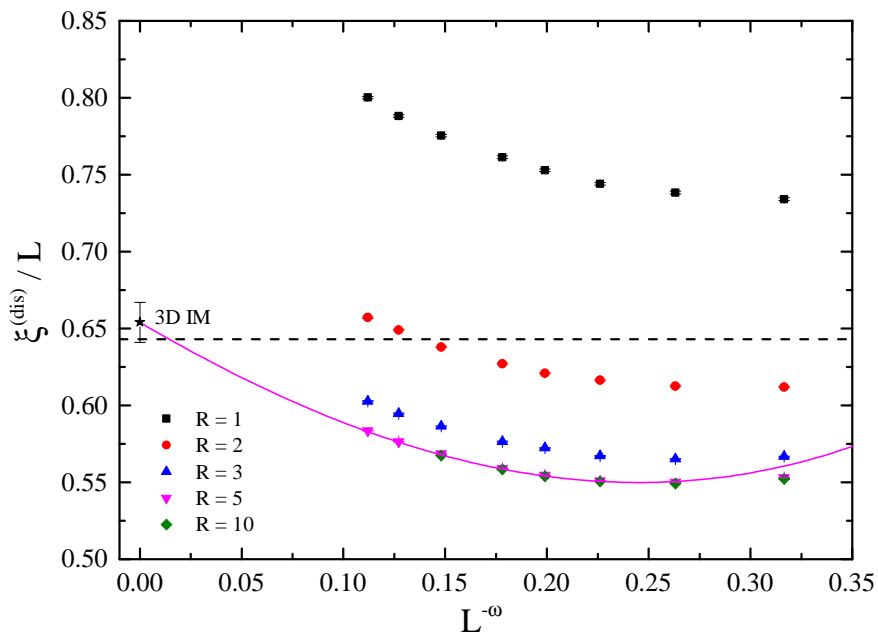

FIG. 1. $\xi^{\text {(dis) }}(L, R) / L$ vs. $L^{-\omega}$ for various $R$ values, as computed in the $D=5 \mathrm{RFIM}$. The value of the corrections to scaling exponent $\omega$ corresponds to the pure Ising model in three spatial dimensions, see Table I] (the value from Ref. 44] is so accurate that we took their central value as numerically exact). The dashed horizontal line corresponds to the value for $\xi / L$, also shown in Table I] The continuous line is a fit to our $R=5$ data (see text for details). The extrapolation to $L=\infty$ obtained from the fit is compatible with the pure Ising model value, as predicted by supersymmetry.

that we aim to recover from the $D$ dimensional RFIM. We shall need as well the value of the leading corrections to scaling exponent $\omega$ ); the analysis we present is done using the exponent $\omega$ given by dimensional reduction, which is not far from the one computed in the large-scale simulations at $D=5[26$.

First, we consider the dimensionless ratio $\xi^{(\mathrm{dis})}(L, R) / L$ in Fig. 1. Our first task, recall Eq. (4), is to extract the large- $R$ limit. The good news is that we expect this limit to be reached exponentially in $R$ and uniformly in $L$ [45]. In fact, the comparison of our numerical results for $R=5$ and 10 suggests that (within our statistical accuracy) $R=5$ is large enough. Therefore, we focus the analysis on $R=5$, where we reach our largest $L$ value, namely $L=14$. As it is clear from Fig. 1, our data are accurate enough to resolve corrections to scaling. Furthermore, the non-monotonic $L$-evolution of $\xi^{\text {(dis) }}(L, R=5) / L$ implies that subleading corrections cannot be neglected. Hence, we have attempted to represent these sub-leading corrections in an effective way by means of a fit to a polynomial

TABLE I. Universal quantities as computed in the pure Ising model at two and three spatial dimensions. The somewhat controversial situation with the corrections to scaling exponent $\omega$ in two dimensions is discussed in Appendix D

\begin{tabular}{clll}
\hline \hline$D-2$ & \multicolumn{1}{c}{$\xi / L$} & \multicolumn{1}{c}{$U_{4}$} & \multicolumn{1}{c}{$\omega$} \\
2 & $0.9050488 \ldots[42]$ & $1.16793 \ldots[4]$ & 1.75 \\
3 & $0.6431(1)[43$ & $1.6036(1)[43$ & $0.82966(9)[44]$ \\
\hline \hline
\end{tabular}

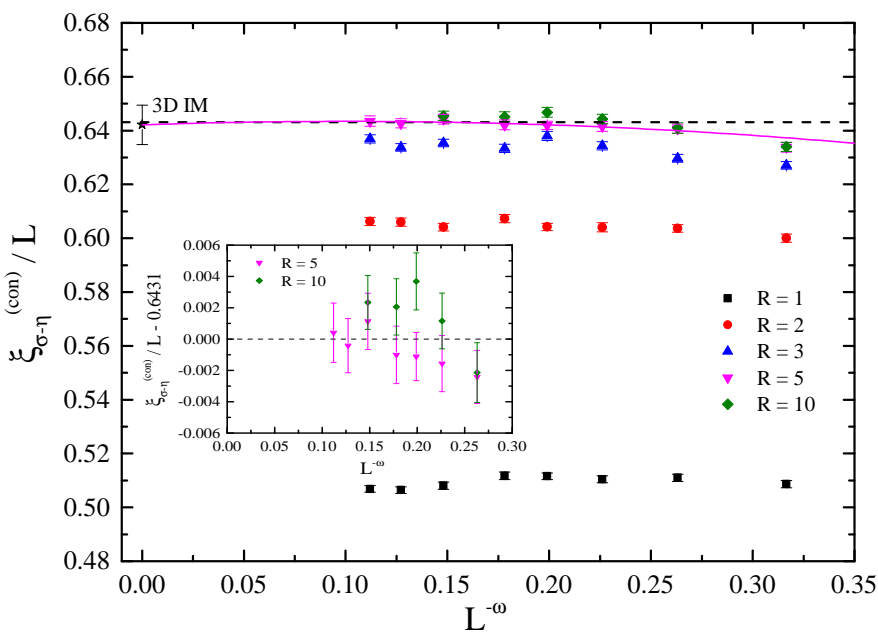

FIG. 2. As in Fig. 1 but for the $\xi_{\sigma-\eta}^{(\text {con })}(L, R) / L$ data, as computed in the $D=5$ RFIM. The agreement of the $L=$ $\infty$ extrapolation with the value of $\xi / L$ from the pure Ising model is a direct confirmation of the supersymmetric Ward identity, see Appendix B. Inset: Zoom of main panel data corresponding to $R=5,10$, and $L>4$. For the sake of clarity, in the vertical axis, we have subtracted the value of the pure Ising model (see also Table I).

in $L^{-\omega}$. We have included in the fit only data with $L \geq L_{\min }$. We have attempted to keep both $L_{\min }$ and the order of the polynomial as low as possible. We find a fair fit $\left(\chi^{2} /\right.$ dof $=3.24 / 2, p$-value $\left.=20 \%\right)$ with a cubic polynomial and $L_{\min }=6$. The corresponding extrapolation to $L=\infty$ is

$$
\lim _{L \rightarrow \infty}\left(\lim _{R \rightarrow \infty} \frac{\xi^{(\mathrm{dis})}(L, R)}{L}\right)=0.654(13),
$$

which is statistically compatible to the three-dimensional result in Table I. Hence, our first check of supersymmetry has been passed. The strength of this check is quantified by our $2 \%$ accuracy.

The analysis of $\xi_{\sigma-\eta}^{(\mathrm{con})}(L, R) / L$, see Fig. 2 is carried out along the same lines. We find a good fit $\left(\chi^{2} /\right.$ dof $=$ $0.63 / 3, p$-value $=89 \%$ ) with a quadratic polynomial in $L^{-\omega}$ and $L_{\min }=6$. The corresponding extrapolation to $L=\infty$ is

$$
\lim _{L \rightarrow \infty}\left(\lim _{R \rightarrow \infty} \frac{\xi_{\sigma-\eta}^{(\mathrm{con})}(L, R)}{L}\right)=0.642(7) .
$$

It follows that we have checked supersymmetry to a $1 \%$ accuracy.

Our $U_{4}(L, R)$ data, see Fig. 3. can be analyzed in a similar vein. We find a fair fit $\left(\chi^{2} /\right.$ dof $=6.85 / 4, p$ value $=14 \%$ ) with a quadratic polynomial in $L^{-\omega}$ and $L_{\min }=5$. The corresponding extrapolation to $L=\infty$ is

$$
\lim _{L \rightarrow \infty}\left(\lim _{R \rightarrow \infty} U_{4}(L, R)\right)=1.604(3),
$$

again compatible with the three-dimensional pure Ising model value (Table I). Supersymmetry is checked to the $0.2 \%$ level, this time. 


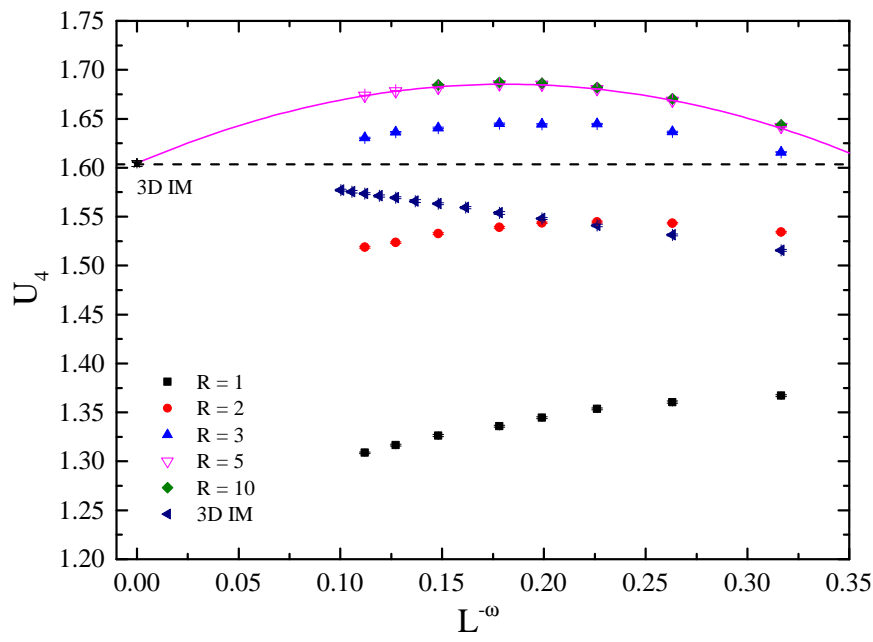

FIG. 3. As in Fig. 1 but for the $U_{4}(L, R)$ data, as computed in the $D=5$ RFIM. For comparison, we also show data for the pure Ising model in three spatial dimensions. Corrections to scaling in the pure model are of similar size (but opposite sign) to those of the large $R$ limit for the RFIM at $D=5$.

Finally, as a comparison, we show our data for the 4D RFIM Ising model in Fig 4 . Even after carrying out the double limit $L \rightarrow \infty$ and $R \rightarrow \infty$, all three dimensionless quantities differ from their values in the $2 \mathrm{D}$ pure Ising ferromagnet. Although this is hardly a surprise (recall, for instance, exponents $\eta$ and $\bar{\eta}$ [25]), the discrepancy is at least at the $10 \%$ level.

Conclusions. - The finding of supersymmetry and dimensional reduction in the RFIM is, arguably, one of the most surprising results in Theoretical Physics. Here, thanks to state-of-the-art numerical techniques, we have carried out a precision test of supersymmetry. Although supersymmetry is clearly broken at $D=4$, the $D=5$ RFIM is supersymmetric with good accuracy. Hence, the Scenario 1 in the Introduction is plainly discarded.

The only remaining contenders are Scenarios 2 and 3. Exponent $\omega$ might help to settle the question. In the $\epsilon$ expansion $(\epsilon=6-D)$ we find at least two exponents: $\omega_{\mathrm{DR}}=\epsilon+\mathcal{O}\left(\epsilon^{2}\right)$ (obtained through dimensional reduction) and $\omega_{\mathrm{NS}}=2+\mathcal{O}\left(\epsilon^{2}\right)$ (due to irrelevant nonsupersymmetric operators). The large value of $\omega$ found here and in Ref. [26] (the values for $\omega(D)$ are in Appendix C), agrees with dimensional reduction and favors Scenario 2. Indeed, in Scenario 3 supersymmetry is broken only for space dimension $D<D_{\text {int }}$, suggesting a much smaller value $\omega(D=5) \sim D_{\text {int }}-D \approx 0.1$. However, further studies are needed to resolve this delicate issue.

\section{ACKNOWLEDGMENTS}

We acknowledge partial financial support from Ministerio de Economía, Industria y Competitividad (MINECO, Spain) through Grant No. FIS2015-65078$\mathrm{C} 2$, and from the European Research Council (ERC) un-

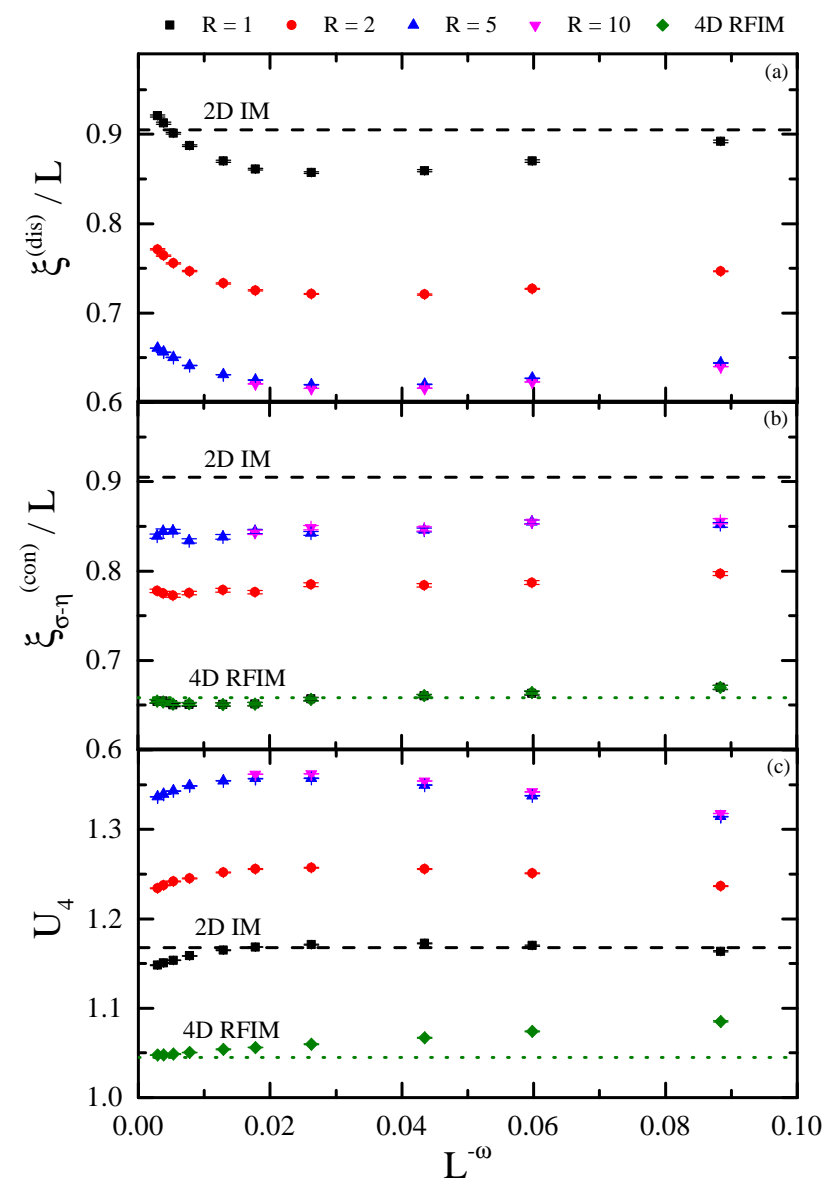

FIG. 4. Dimensionless quantities $\xi^{(\mathrm{dis})}(L, R) / L \quad(\mathbf{a})$, $\xi_{\sigma-\eta}^{(\text {con })}(L, R) / L(\mathbf{b})$ and $U_{4}(L, R)(\mathbf{c})$ vs. $L^{-\omega}$ as computed in the $D=4$ RFIM. We set $\omega=1.75$ from Table [ We show the corresponding universal values for the $2 \mathrm{D}$ pure Ising model (black dashed lines). Note that for $R=1$ there are two natural ways of computing $U_{4}$. One way (black squares) is averaging over a co-dimension two manifold [this is the natural way for a supersymmetry check, recall Eq. [6]]. The other way, which is the natural one when studying the $D=4$ RFIM per se, is averaging over the full four-dimensional lattice (green diamonds). Clearly, the two choices differ, both at finite $L$ and in the large- $L$ limit. Instead, for $\xi_{\sigma-\eta}^{\text {(con) }}(L, R) / L$ these two kinds of spatial-averaging coincide by construction. The horizontal green dotted lines are the large- $L$ limit, as obtained for the $D=4$ RFIM [25].

der the European Union's Horizon 2020 research and innovation program (Grant No. 694925). N. G. F. and M. P. were supported by a Royal Society International Exchanges Scheme 2016/R1. 


\section{Appendix A: Finite volume supersymmetry}

In the case of RFIM in the Landau-Ginsburg form, it is well known that we can neglect the thermal fluctuations near the critical temperature and the model becomes equivalent to a stochastic differential equation. Under the approximation of uniqueness of the solution, we arrive to a supersymmetric field theory. In this theory we can define the superfield $\Phi(X)$ as function of the superposition $X=x \oplus \theta$,

$$
\Phi(X)=\phi(x)+\bar{\theta} \psi(x)+\bar{\psi}(x) \theta+\bar{\theta} \theta \lambda(x),
$$

where $\theta$ is a complex anticommuting quantity, $\phi(x)$ is the original field and $\psi(x)$ and $\lambda(x)$ are auxiliary fields, whose correlations functions are related to the response functions. For instance, in the supersymmetric formulation the connected propagator $C_{x y}^{(\text {con })}$ corresponds to the propagator of the fermionic field $\langle\bar{\psi}(x) \psi(y)\rangle$, while the disconnected propagator $C_{x y}^{(\mathrm{dis})}$ corresponds to the propagator for the bosonic field $\langle\phi(x) \phi(y)\rangle$.

In the infinite volume limit, the theory is invariant under the supergroup $O(D \mid 2)$ which implies that the correlation functions are functions of the superdistances. In particular, the correlation function $\langle\Phi(X) \Phi(Y)\rangle$ is a function of

$$
(X-Y)^{2}=r^{2}+\left(\theta_{x}-\theta_{y}\right)\left(\bar{\theta}_{x}-\bar{\theta}_{y}\right),
$$

where $r^{2}$ is the (squared) Euclidean distance between points $x$ and $y$ in the $D$-dimensional space:

$$
\langle\Phi(x) \Phi(y)\rangle=\langle\phi(x) \phi(y)\rangle+\bar{\theta} \theta\langle\bar{\psi}(x) \psi(y)\rangle=F(Z)
$$

where $Z=(X-Y)^{2}$. By Taylor expanding both sides of Eq. (A3) in powers of $\bar{\theta} \theta$ we conclude that

$$
F(Z)=F\left(r^{2}\right)+\bar{\theta} \theta F^{\prime}\left(r^{2}\right),
$$

because all higher powers of $\bar{\theta} \theta$ vanish. We readily obtain the Ward identity [38]

$$
\langle\bar{\psi}(x) \psi(y)\rangle=-\frac{\mathrm{d}\langle\phi(x) \phi(y)\rangle}{\mathrm{d} r^{2}} .
$$

We note that Eq. A5 implies for the RFIM in a infinite lattice that

$$
C_{r}^{(\text {con })}=-\mathcal{Z}_{2} \frac{\mathrm{d}}{\mathrm{d} r^{2}} C_{r}^{(\text {dis })},
$$

where large $r$ and $\xi$ are assumed ( $\xi$ is the correlation length), so that $D$-dimensional rotational invariance is restored, and $\mathcal{Z}_{2}$ is a position-independent (therefore, irrelevant for us) constant 46. These relations A3 A6 lead to a bunch of Ward identities among various correlation functions. One also finds that the probability distribution of the $\phi$ field on a $d \equiv D-2$-dimensional hyperplane is the same of the dimensional reduced theory.
However, in a finite volume rotational invariance is broken so that supersymmetry and dimensional reduction are lost. Fortunately close examination of the argument shows that we do not need the full $O(D \mid 2)$ supersymmetry, but the $O(2 \mid 2)$ supersymmetry is enough in order to have dimensional reduction. In order to recover the $O(2 \mid 2)$ supersymmetry, the system size needs to be infinite only in the remaining two dimensions.

Our choice (see main text) is to stay in a system of linear size $L$ in $d$ directions and of size $L R$ in two directions. At the end we need to consider the limit $R \rightarrow \infty$ in order to have supersymmetry and dimensional reduction. Let us write the $D$ dimensional coordinates $\mathbf{r}$ as $(\mathbf{x}, \mathbf{u})$, where $x$ is $d$-dimensional and $u$ is two dimensional. We can write

$$
X=\mathbf{r} \oplus \theta=\mathbf{x} \oplus \mathbf{u} \oplus \theta .
$$

The $O(2 \mid 2)$ supersymmetry acts on the two-dimensional subspace, labeled by coordinates $\mathbf{u} \oplus \theta$, that becomes infinite in the $R \rightarrow \infty$ limit. Dimensional reduction gives informations only on the probability distribution on fields on the hyperplanes at fixed $\mathbf{u}$ that have volume $L^{d}$.

Supersymmetry does not give us information on the behaviour of the correlations function of fields whose $\mathbf{u}$ is different, unless we stay at distances much smaller than $L$, where $2+d$ rotational invariance is recovered. It connects however responce functions at different $\mathbf{u}$ with the correlations functions at fixed $\mathbf{u}$, as we shall see below.

\section{Appendix B: The Ward Identity and its consequences}

As explained above (see also main text), we shall be considering points in the five-dimensional lattice, $\mathbf{r}=$ $(\mathbf{x}, \mathbf{u})$ where $\mathbf{x}=(x, y, z)$ refers to the first three cartesian coordinates, while $\mathbf{u}=(t, u)$. In a similar vein, for the $D=4$ case, we split $\mathbf{r}=(x, y, z, t)=(\mathbf{x}, \mathbf{u})$ as $\mathbf{x}=$ $(x, y)$ and $\mathbf{u}=(z, t)$. The (squared) Euclidean distance between two points in the $D$ dimensional lattice will be named $r^{2}=\mathbf{x}^{2}+\rho^{2}$ (in $D=5, \rho^{2}=t^{2}+u^{2}$, while in $D=4$ we have $\left.\rho^{2}=z^{2}+t^{2}\right)$.

In the finite $L$ case we only have a $O(2 \mid 2)$ supersymmetry. Therefore, instead of the Ward identities corresponding to $O(D \mid 2)$, see Eqs. A2 A6), the Bosonic and Fermionic propagators are now related through a $O(2 \mid 2)$ Ward identity that tells us that

$$
C_{\mathbf{x}, \mathbf{u}}^{(\text {con })}=-\mathcal{Z}_{2} \frac{\mathrm{d}}{\mathrm{d} \rho^{2}} C_{\mathbf{x}, \mathbf{u}}^{(\mathrm{dis})} .
$$

In our geometry, we only have the full $D$-dimensional rotational symmetry for $\mathrm{x}^{2} \ll L^{2}$. Instead, in the limit of a large aspect ratio, $R \rightarrow \infty$, we have two-dimensional rotational symmetry (for the $\mathbf{u}$ variables) for any $\mathbf{x}$. Thus, we expect the two correlation functions $C_{\mathbf{x}, \mathbf{u}}^{(\text {dis })}$ and $C_{\mathbf{x}, \mathbf{u}}^{(\text {con })}$ to be functions of

$$
g(\mathbf{x})+\rho^{2},
$$


where $g(\mathbf{x})$ is some function of the $d$-dimensional coordinates that reduces to the $d$-dimensional Euclidean distance $\mathbf{x}^{2}$ in the limit $\mathbf{x}^{2} \ll L^{2}$ [a simple possibility in $D=5$ would be $g(\mathbf{x})=L^{2} \pi^{-2}\left(\sin ^{2} \pi x / L+\sin ^{2} \pi y / L+\right.$ $\left.\left.\sin ^{2} \pi z / L\right)\right]$.

Let us now consider the $\mathbf{u}$-averaged correlation function

$$
K_{\mathbf{x}_{1} ; \mathbf{x}_{2}}=\sum_{\mathbf{u}} C_{\mathbf{x}_{1}, \mathbf{0} ; \mathbf{x}_{2}, \mathbf{u}}^{(\mathrm{con})}
$$

The $D=5$ reasoning goes as follows (the $D=4$ case is analogous):

$$
K_{\mathbf{x}_{1} ; \mathbf{x}_{2}} \approx \iint_{-\infty}^{\infty} \mathrm{d} t \mathrm{~d} u C_{\mathbf{x}_{1}, 0,0 ; \mathbf{x}_{2}, t, u}^{(\mathrm{con})}
$$

We now introduce polar coordinates in the $(t, u)$ plane, $t=\rho \cos \varphi$ and $u=\rho \sin \varphi$ :

$$
K_{\mathbf{x}_{1} ; \mathbf{x}_{2}} \approx \pi \int_{0}^{\infty} \mathrm{d} \rho^{2} C_{\mathbf{x}_{1}, 0,0 ; \mathbf{x}_{2}, \rho, 0}^{(\mathrm{con})} .
$$

Our next step, will be using the Ward identity (B1):

$$
K_{\mathbf{x}_{1} ; \mathbf{x}_{2}} \approx \pi \mathcal{Z}_{2} \int_{0}^{\infty} \mathrm{d} \rho^{2}\left[-\frac{\mathrm{d}}{\mathrm{d} \rho^{2}} C_{\mathbf{x}_{1}, 0,0 ; \mathbf{x}_{2}, \rho, 0}^{(\mathrm{dis})}\right],
$$

and thus, we finally get

$$
K_{\mathbf{x}_{1} ; \mathbf{x}_{2}} \approx \pi \mathcal{Z}_{2} C_{\mathbf{x}_{1}, 0,0 ; \mathbf{x}_{2}, 0,0}^{(\text {dis })}
$$

Note that, because we shall be taking the limit of large $R$ at fixed $L$, the gap in the transfer matrix scales as $1 / L$. Therefore, the correlation function $C_{\mathbf{x}_{1}, 0,0 ; \mathbf{x}_{2}, \rho, 0}^{(\text {dis })}$ decays exponentially in $\rho$ (for any $L$ ), so the convergence of the two-dimensional integrals in Eqs. (B4)-(B6) poses no problems.

Hence, in the large- $R$ limit, the second-moment correlation length $\xi_{\sigma-\eta}^{(\text {con })}$ is predicted to coincide with the one obtained from the disconnected propagator. The prediction holds to a high accuracy in the RFIM in $D=5$, but certainly not in $D=4$ (see Fig. 4 in the main part).

Let us conclude this section by explainig our naming $\xi_{\sigma-\eta}^{\text {(con })}$ to the correlation length extracted from the $K$ propagator, which stems from the way it is computed. Indeed, the Fluctuation-Dissipation relations for Gaussian random-fields [24] suggest a simple way to compute the $K_{\mathbf{x}_{1} ; \mathbf{x}_{2}}$ propagator. Let

$$
\sigma(\mathbf{x})=\sum_{\mathbf{u}} S_{\mathbf{x}, \mathbf{u}}, \eta(\mathbf{x})=\sum_{\mathbf{u}} h_{\mathbf{x}, \mathbf{u}},
$$

then

$$
K_{\mathbf{x}_{1} ; \mathbf{x}_{2}}=\frac{1}{R^{2} L^{2}} \overline{\left\langle\sigma\left(\mathbf{x}_{\mathbf{1}}\right) \eta\left(\mathbf{x}_{\mathbf{2}}\right)\right\rangle} .
$$

Of course, $\mathbf{x}_{\mathbf{1}}$ and $\mathbf{x}_{\mathbf{2}}$ might be interchanged, so it is better to average over the two orderings.

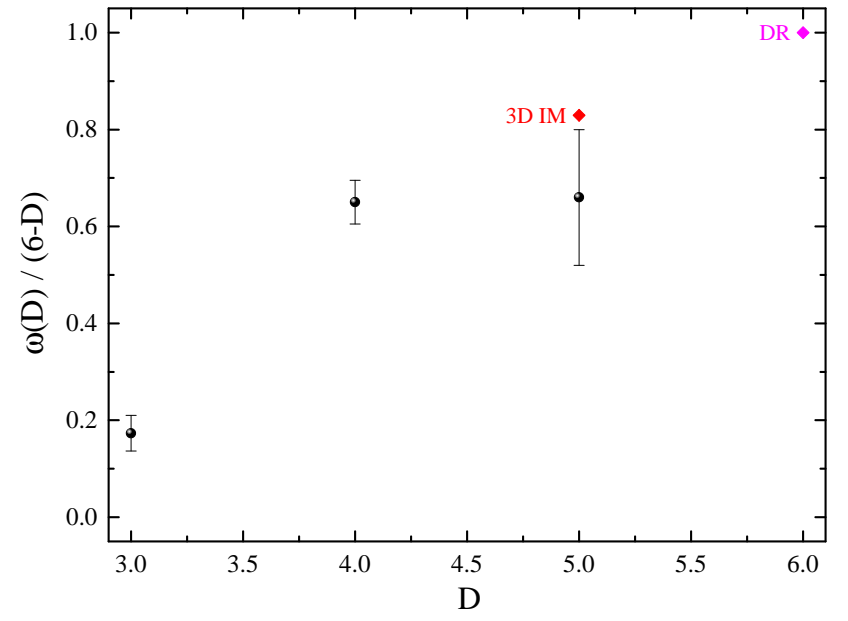

FIG. 5. The corrections to scaling exponent $\omega$, as computed from the RFIM in $D=3[23, D=4$ [25] and $D=5$ [26] in units of $6-D$ versus the space dimension. If we explicitly assume dimensional reduction (DR), we also have an exceedingly more accurate result for $D=5$ (from the threedimensional pure Ising model (3D IM) 44]) and an exact result at $D=6$.

\section{Appendix C: Exponent $\omega$ for the RFIM: the smoking gun?}

As discussed in the conclusions of the main part, dimensional reduction suggests that $\omega(D)=\epsilon+\mathcal{O}\left(\epsilon^{2}\right)$, with $\epsilon=6-D$. Indeed, Fig. 5 strongly suggests that the dimensional-reduction prediction is sensible, because $\omega(D) /(D-6)$ seems a very smooth function of $D$. We do not find any indication for a zero of $\omega(D)$ near $D=5$. It is our impression that such a zero, which we do not see, would be a direct prediction of the Scenario 3 discussed in the main paper.

\section{Appendix D: Exponent $\omega$ for the pure Ising model in $D=2$}

Paradoxically, it is not trivial to determine the scaling corrections exponent $\omega$ in the $D=2$ pure Ising model, which is one of the best known models in Statistical Mechanics.

The difficulty lies in that the leading correction to scaling seems to have a somewhat unusual origin. Consider, for instance, the magnetic susceptibility $\chi$ as computed at the critical point for a system of linear dimension $L$. It is expected to scale as

$$
\chi \sim A L^{2-\eta}+C,
$$

where $\eta=1 / 4$ is the anomalous dimension, $A$ is a scaling amplitude and $C$ is a constant term due to the analytic part of the free-energy density. Eq. (D1) can be cast as well in the typical form for scaling-corrections studies (see, e.g., Ref. [37]):

$$
\chi \sim L^{2-\eta}\left(A+C L^{-\omega}\right) \quad, \quad \omega=2-\eta=7 / 4 .
$$


However, this exponent $\omega=7 / 4$ is not related to any irrelevant operator, but to the analytic part of the freeenergy. Hence, the reasoning leading us to Eq. (D2) makes sense only if the $\omega$ exponents arising from all the irrelevant operators are larger than $7 / 4$. Only under this assumption the leading corrections to scaling would be given by Eq. (D2).

Now, it is well known that an operator associated to the dilution for the q-Potts models in $D=2$ (the $q=2$ Potts model is the Ising model) has dimension $10 / 3$ and then $\omega=-(D-10 / 3)=4 / 3$ [47. According to the discussion above, the leading corrections to scaling would then be given by $\omega=4 / 3$, rather than $7 / 4$. However, we think this is not the case, due to a number of theoretical and numerical reasons:

- This dilution operator is outside of the main Kac table of operators for the Ising model. Thus it is not produced by other operators (susch as the Identity, spin or energy operators) and then it is expected that this operator does not contribute to the corrections to scaling. Note that, on the contrary, the operator is inside the Kac table for other Conformal Field Theories (CFT), such as the 3Potts model [48, for instance. In fact, in the limit $q \rightarrow 4$, the critical points for Potts and the tricritical Potts (which corresponds to the dilution fixed point) merge and, indeed, the dilution operator has a dimension 2 in this limit. It is one example for which one finds $\omega=0$.

- The above analytical reasoning was confirmed in Ref. 49]. which considered (numerically) various extension of the Ising model (antiferromagnetic Ising model in a magnetic field and the BlumeCapel model). The exponent $\omega=4 / 3$ was not found in any of these models (rather, a correction $\omega \simeq 2$ was identified). Indeed, the authors of Ref. 49] concluded that the dilution contribution to the correction to scaling is indeed given by an exponent $\omega=4 / 3$, but with amplitudes proportional to $(q-2)$ and thus is absent for the Ising model, in agreement with CFT predictions. This scenario was supported by simulations of the random-cluster model for $q$ close to 2 .

- A recent, very-high accuracy simulation [50] found again $\omega=7 / 4$.
[1] G. Parisi and N. Sourlas, Phys. Rev. Lett. 43, 744 (1979)

[2] Y. Imry and S.-k. Ma, Phys. Rev. Lett. 35, 1399 (1975)

[3] T. Nattermann, in Spin glasses and random fields, edited by A. P. Young (World Scientific, Singapore, 1998).

[4] D. P. Belanger, in Spin Glasses and Random Fields, edited by A. P. Young (World Scientific, Singapore, 1998).

[5] A. Aharony, Y. Imry, and S.-k. Ma, Phys. Rev. Lett. 37, 1364 (1976)

[6] A. P. Young, Journal of Physics C: Solid State Physics 10, L257 (1977)

[7] J. L. Cardy, Physics Letters B 125, 470 (1983).

[8] G. Parisi and N. Sourlas, Phys. Rev. Lett. 46, 871 (1981)

[9] D. C. Brydges and J. Z. Imbrie, Annals of Mathematics 158, 1019 (2003)

[10] J. Z. Imbrie, Annales Henri Poincaré 4, 445 (2003).

[11] J. L. Cardy, "Lecture on branched polymers and dimensional reduction," (2003), unpublished, arXiv:condmat/0302495

[12] J. Z. Imbrie, Phys. Rev. Lett. 53, 1747 (1984).

[13] J. Bricmont and A. Kupiainen, Phys. Rev. Lett. 59, 1829 (1987)

[14] G. Parisi, Field Theory, Disorder and Simulations (World Scientific, 1994).

[15] G. Parisi and N. Sourlas, Phys. Rev. Lett. 89, 257204 (2002)

[16] E. Brézin and C. De Dominicis, EPL (Europhysics Letters) 44, 13 (1998)

[17] E. Brézin and C. De Dominicis, Eur. Phys. J. B 19, 467 (2001)

[18] G. Parisi and V. Dotsenko, Journal of Physics A: Mathematical and General 25, 3143 (1992)

[19] V. S. Dotsenko, Journal of Statistical Mechanics: Theory and Experiment 2007, P09005 (2007).

[20] M. Tissier and G. Tarjus, Phys. Rev. Lett. 107, 041601 (2011)

[21] M. Tissier and G. Tarjus, Phys. Rev. B 85, 104203 (2012).

[22] G. Tarjus, I. Balog, and M. Tissier, EPL (Europhysics Letters) 103, 61001 (2013)

[23] N. G. Fytas and V. Martín-Mayor, Phys. Rev. Lett. 110, 227201 (2013)

[24] N. G. Fytas and V. Martín-Mayor, Phys. Rev. E 93, 063308 (2016)

[25] N. G. Fytas, V. Martín-Mayor, M. Picco, and N. Sourlas, Phys. Rev. Lett. 116, 227201 (2016)

[26] N. G. Fytas, V. Martín-Mayor, M. Picco, and N. Sourlas, Phys. Rev. E 95, 042117 (2017).

[27] S. Hikami, "Dimensional reduction by conformal bootstrap," (2018), unpublished, arXiv:1801.09052.

[28] N. G. Fytas, V. Martín-Mayor, M. Picco, and N. Sourlas, Journal of Statistical Physics 172, 665 (2018).

[29] J.-C. Anglès d'Auriac, M. Preissmann, and R. Rammal, J. Physique Lett. 46, 173 (1985)

[30] A. T. Ogielski, Phys. Rev. Lett. 57, 1251 (1986)

[31] A. A. Middleton, Phys. Rev. Lett. 88, 017202 (2001)

[32] A. A. Middleton and D. S. Fisher, Phys. Rev. B 65, 134411 (2002)

[33] A. A. Middleton, "Scaling, domains, and states in the four-dimensional random field ising magnet," (2002), preprint, arXiv:cond-mat/0208182

[34] J. Villain, Phys. Rev. Lett. 52, 1543 (1984)

[35] A. J. Bray and M. A. Moore, Phys. Rev. B 31, 631 (1985).

[36] D. S. Fisher and D. A. Huse, Phys. Rev. Lett. 56, 1601 (1986)

[37] D. J. Amit and V. Martín-Mayor, Field Theory, the 
Renormalization Group and Critical Phenomena, 3rd ed. (World Scientific, Singapore, 2005).

[38] G. Parisi and N. Sourlas, Nuclear Physics B 206, 321 (1982)

[39] A. Klein, L. Landau, and J. Perez, Communications in Mathematical Physics 94, 459 (1984)

[40] J. L. Cardy, Physica D: Nonlinear Phenomena 15, 123 (1985).

[41] We introduce the Fourier transform in $(D-2)$ dimensions, $\hat{K}(\mathbf{k})=\sum_{\mathbf{x}_{1}, \mathbf{x}_{2}} \mathrm{e}^{\mathrm{i}\left(\mathbf{x}_{1}-\mathbf{x}_{2}\right) \cdot \mathbf{k}} K_{\mathbf{x}_{1}, \mathbf{x}_{2}} / L^{D-2}$ and compute $\xi_{\sigma-\eta}^{(\text {con })}=\left[\left(\hat{K}(\mathbf{0})-\hat{K}\left(\mathbf{k}_{\min }\right) / \hat{K}\left(\mathbf{k}_{\min }\right)\right]^{1 / 2} /(2 \sin \pi / L)\right.$. For an extended discussion of the second-moment correlation length see, for instance, Ref. 37.

[42] J. Salas and A. D. Sokal, Journal of Statistical Physics 98, 551 (2000)

[43] M. Hasenbusch, Phys. Rev. B 82, 174433 (2010)

[44] F. Kos, D. Poland, D. Simmons-Duffin, and A. Vichi,
Journal of High Energy Physics 08, 36 (2016).

[45] Because we shall be taking the limit of large $R$ at fixed $L$, the gap in the transfer matrix scales as $1 / L$. Therefore, correlation functions along the $t$ and $u$ axes $(z$ and $t$ axes at $D=4$ ), decay exponentially in $R$, for any $L$.

[46] When combined with the long distance decay of the propagators at the critical point, $C_{r}^{(\text {con })} \sim 1 / r^{D-2+\eta}$ and $C_{r}^{(\text {dis })} \sim 1 / r^{D-4+\bar{\eta}}$, the Ward identity A6 tells us that $\eta=\bar{\eta}$.

[47] B. Nienhuis, Journal of Physics A: Mathematical and General 15, 199 (1982)

[48] V. Dotsenko, Nuclear Physics B 235, 54 (1984)

[49] H. W. J. Blöte and M. P. M. den Nijs, Phys. Rev. B 37, 1766 (1988). [50] H. Shao, W.
$\begin{aligned} & \text { Hondvik, } \\ & \text { Sand }\end{aligned}$ http://science.sciencemag.org/content/352/6282/213.full.pdf 